\title{
CONDUCTA SUICIDA Y PERIODO PERINATAL: ENTRE EL TABÚ Y LA INCOMPRENSIÓN
} SUICIDAL BEHAVIOR AND THE PERINATAL PERIOD: TABOO AND MISUNDERSTANDING

\author{
Susana Al-Halabí', Juan García-Haro², María de la Fe Rodríguez-Muñoz ${ }^{3}$ y \\ Eduardo Fonseca-Pedrero ${ }^{4}$ \\ 'Universidad de Oviedo. ${ }^{2}$ Servicios de Salud del Principado de Asturias. ${ }^{3}$ Universidad Nacional de Educación a Distancia \\ ${ }^{4}$ Universidad de La Rioja
}

El suicidio es la primera causa de muerte de las mujeres durante el periodo perinatal, que comprende desde el embarazo hasta un año después del parto. Hay apoyo empírico suficiente para afirmar que las mujeres embarazadas tienen mayor ideación suicida que su correspondiente grupo de comparación en la población general. A pesar de estos datos, este tipo de problemas no suelen ni prevenirse ni reconocerse adecuadamente. Sin embargo, si las disonancias y dilemas asociados a la maternidad, así como los problemas de salud mental, no se previenen o se abordan adecuadamente, éstos pueden afectar al bienestar de las mujeres, al de sus hijos y al de otros miembros de la familia. Se exponen los factores implicados en la conducta suicida de este grupo de mujeres, así como algunas directrices generales de actuación. Se reclama la necesaria puesta en marcha de estrategias de prevención.

Palabras clave: Conducta suicida, Suicidio, Embarazo, Posparto, Perinatal, Prevención.

Suicide is the leading cause of death for women during the perinatal period, which commences in pregnancy and finishes one year after delivery. Empirical evidence from previous studies shows that pregnant women have greater suicidal ideation than their comparison group in the general population. However, there is a tendency for these problems to be neither prevented nor adequately recognized. Nevertheless, if the dissonances and dilemmas associated with motherhood, as well as mental health problems, are not prevented or adequately addressed, they can affect the well-being of women, their children, and other family members. Risk and protective factors for suicidal behavior in this group of women are discussed, as well as general principles of action. The need for the implementation of prevention strategies is highlighted.

Key words: Suicidal behavior, Suicide, Pregnancy, Postpartum, Perinatal, Prevention.

11

Nadie te dice que esto puede pasar, y cuando pasa, estás tan perdida y todo es tan oscuro que ni siquiera sabes que puedes pedir ayuda". Posiblemente no sea un error pensar que la mayoría de los profesionales de la psicología (y de otras profesiones sanitarias) desconoce que el suicidio es la primera causa de muerte de las mujeres durante el periodo perinatal, que comprende desde el embarazo hasta un año después del parto (Enătescu et al., 2020; Gelaye et al., 2017; Knight et al., 2019).

Seguramente el interés que suscita este tema se deba, en parte, a la tristeza y perplejidad que despiertan las noticias sobre los suicidios de las (recientes o futuras) madres junto con la presencia de otros fenómenos como el neonaticidio o el infanticidio, sucesos éstos raros, pero -al igual que el suicidio- complejos y con profundas raíces psicológicas (Al-Halabí, 2019; Al-Halabí et al., 2019; Al-Halabí y García-Haro, 2021; Al-Halabí et al., 2021). Sin embargo, tras varias décadas de investigación aún no tenemos una narrativa coherente que permita comprender la compleja asociación entre maternidad y suicidio (Fisher, 2016). Sí parece claro que los mitos acerca del amor inmediato hacia los hijos recién nacidos suponen una importante barrera para que las mujeres pidan ayuda o consigan los

Recibido: 1 marzo 2021 - Aceptado: 6 mayo 2021

Correspondencia: Susana Al-Halabí. Facultad de Psicología.

Plaza Feijoo, s/n, despacho 230.33003 Oviedo. España.

E-mail: alsusana@uniovi.es recursos que necesitaba para afrontar una crisis suicida. A pesar de lo contundente que resulta el dato que abre este artículo, apenas se han establecido intervenciones preventivas específicas para el abordaje de este problema, cuyas consecuencias son devastadoras, tanto para los bebés que pierden a sus madres, como para las familias, cuyo sufrimiento desborda los parámetros habituales acerca de los costes sociales y sanitarios (Al-Halabí et al., 2019).

El objetivo de este artículo es claro: sensibilizar acerca del tabú que existe, tanto en la sociedad como entre los profesionales, sobre la relación entre el suicidio y la maternidad, concienciar sobre este problema aportando información breve pero relevante y señalar la necesidad de intervenciones preventivas para la conducta suicida durante este periodo de la vida de las mujeres.

\section{¿QUÉ SABEMOS ACERCA DE LA CONDUCTA SUICIDA DURANTE EL PERIODO PERINATAL?}

La literatura científica sobre este tema no es muy prolija. Nótese aquí que, en nuestro país, el Instituto Nacional de Estadística no cuenta con datos desglosados para conocer el alcance de este fenómeno. La escasez y variabilidad de los datos puede deberse a la falta de consenso que aún hoy persiste en la nomenclatura de la conducta suicida (para tal asunto derivamos al lector a fuentes de gran interés como, por ejemplo, De Beurs et al., 2020; Fonseca-Pedrero et al., 2020; Hill et al., 2020; Leather et al., 2020; Silverman, 2016; Silverman y Berman, 2017; Silverman y DeLeo, 2016; van Mens et al., 2020; Turecki et al., 2019). Hace ya casi dos décadas que Silverman (2006) adver- 
tía que, dado que el término 'tentativa de suicidio' puede significar tantas cosas potencialmente diferentes, corre el riesgo de no significar absolutamente nada. Desde entonces hemos avanzado, no cabe duda, pero aún hoy seguimos anclados en ese problema (Berman y Silverman, 2017). En palabras de Rendueles (2018) "hay un saber sobre el suicidio que se limita a lo artesanal". Luego, más ciencia y más con-ciencia es necesaria en este campo.

En el caso de las mujeres en el periodo perinatal, todo se vuelve aun más complejo, ya que junto a experiencias tan elementales como el malestar y el cansancio, se unen los cambios en el estado de ánimo y otros componentes habituales de la depresión que se pueden vivir como características propias del embarazo y el posparto (Rodríguez-Muñoz et al., 2021). Y también puede suceder lo contrario: que características propias del embarazo y el posparto se vivan como indicios de un trastorno mental incipiente, lo que podría dar lugar a falsos diagnósticos, a incrementar el estigma y a desactivar los propios recursos de solución de las mujeres y sus familias (por supuesto, hay mujeres que no experimentan ninguno de esto problemas y disfrutan de este periodo de sus vidas o lo viven con total normalidad). Esta circunstancia hace que se requiera mayor pericia, sensibilidad y formación por parte del profesional de la psicología para poder establecer con claridad y precisión de qué estamos hablando o, mejor dicho, de qué están hablando o qué están experimentando las mujeres que se encuentran en una situación en la que, para el desconcierto de sus personas más cercanas, empiezan a pensar que "sus familias y sus bebés estarían mejor sin ellas" (Rodríguez-Muñoz, 2019). El suicidio no es algo que ocurra en el vacío, sino que tiene lugar en un contexto específico y en unas circunstancias determinadas de la vida de la (reciente o futura) madre en la que la desesperanza y el sufrimiento cobran especial relevancia. El suicido es un fenómeno multifactorial, plural, interactivo, dinámico y contextual-existencial, lo que se traduce en que, para cada mujer, existen diversas configuraciones problemáticas que pueden estar en la base de los problemas o las dificultades con su maternidad (AlHalabí y García Haro, 2021 ; García-Haro et al., 2018). En el caso que nos ocupa, como en todos los problemas de salud mental, están presentes diversos factores biológicos, psicológicos, sociales y culturales, así como la valencia psicológica que la maternidad tenga para cada mujer. Parece razonable pensar que el "valor" de la maternidad no es "absoluto" ni similar para todas las mujeres ni en todas las culturas, sino que, lejos de la idea de que se trata de un periodo de júbilo y de bienestar emocional, dicho "valor" dependerá -en buena medida- de los valores y el significado que tenga para cada mujer, de su situación personal y familiar, del apoyo recibido por la pareja, de su experiencia previa, del apoyo social disponible, de la presencia de problemas de salud, de los recursos económicos disponibles, de sus expectativas sobre cómo será capaz de afrontar sus nuevas obligaciones y la importancia que en su visión del mundo y de ella misma tienen éstas, etc. (Al-Halabí et al., 2019; RodríguezMuñoz, 2019).

\section{EL MITO DE LA MATERNIDAD EN EL PROCESO DE CONSTRUCCIÓN DE LA IDENTIDAD FEMENINA}

La maternidad es un hecho (pero también un constructo) que histó- ricamente ha modulado la construcción de la identidad femenina. El embarazo y el parto implican el nacimiento de una nueva identidad, a menudo asociada a significados rígidos en torno a lo que es ser "buena madre" (Paricio del Castillo y Polo Usaola, 2020). Se refiere al conjunto de discursos sociales en torno a la experiencia materna (típicamente como un periodo de realización personal), la felicidad, la crianza, la abnegación, el cuidado, la entrega, la renuncia, etc., de la madre. Aunque son discursos externos, pueden acabar por "interiorizarse" en forma de autoprescripción personal.

Son muchas las tribulaciones que enfrenta una mujer durante el periodo perinatal. Sin afán de exhaustividad, empezaremos por lo más obvio, que son los cambios biológicos, de imagen y de rol. Nos referimos así a los cambios en la imagen corporal, a los cambios en los ritmos biológicos, a la disminución del rendimiento, a la sobrecarga por doble jornada, etc. Es de especial interés mencionar la situación de baja laboral, la cual puede ser vivida por muchas madres como antesala de la pérdida del proyecto profesional y del sentido de continuidad.

El nacimiento de la identidad de madre implica un modo de ser radicalmente nuevo que va más allá de la mera "idea" de saber que se es madre. Se trata de un proceso íntimo de transformación que recorre todas las dimensiones psicológicas de la subjetividad, desde lo corporal hasta los procesos de identidad. Ello supone un esfuerzo de integración (se deja de ser sólo hija para ser hija y madre, se deja de ser sólo pareja para ser pareja y madre, etc.) y de revisión de modelos anteriores (con la propia madre, con la pareja, etc.) que nunca está libre de dificultad, más aún si no existe un vínculo de cuidado por parte de la pareja y la familia hacia la mujer que está gestando esa nueva experiencia e identidad maternal, o -como parece probable en la sociedad moderna- si los modelos de crianza están cada vez menos disponibles. Respecto de la identidad, pueden existir dificultades para integrar las propias decisiones y el proyecto profesional en la narrativa social de "buena madre", lo que puede originar dilemas y crisis. Por otro lado, ese estereotipo puede estar apuntalado por el posicionamiento de los otros significativos (pareja, familia, amigos, compañeros de trabajo, profesionales sanitarios, etc.) y sus ideas prototípicas en torno a la buena crianza, la lactancia, etc. En este sentido, y desde la experiencia clínica, es habitual comprobar que se realiza un envío preferente a salud mental a aquellas madres que manifiestan una alta preocupación por el hecho de sentirse mal ("no tengo motivos") y culpables por si tal malestar tuviera un efecto colateral perjudicial en el bienestar de su bebé. Parece evidente que todas estas dificultades de adaptación y los dilemas de identidad se acompañan de experiencias de malestar, típicamente en forma de ansiedad, tristeza, fatiga, impotencia, frustración, rabia, indecisión, problemas de concentración, etc. Estas manifestaciones, muchas veces comprensibles por el propio estado biológico del embarazo y del posparto, pueden activar en la mujer estrategias de control y evitación experiencial que, más que ayudarles a salir del malestar, configuran un bucle que sumerge aun más a sus protagonistas en un estado de sufrimiento y soledad, a su vez vivido con culpa y vergüenza, en tanto que confirmación de una supuesta incapacidad o fracaso para ser "buena madre". 
Este proceso dinámico de lucha ineficaz contra las emociones y de disonancia con el mandato social de la "buena maternidad" puede llevar a muchas mujeres a una auténtica crisis, acompañada del abandono de las actividades de crianza y hasta del rechazo hacia el propio bebé. Por lo que venimos planteando, lo que se atisba es la existencia de un contexto configurador de problemas psicológicos. Este contexto puede atrapar a las mujeres en unos estados de desesperanza y aflicción tal, que cobran sentido los deseos de muerte y las ideas autolíticas que -como veremos a continuaciónson bastante frecuentes en el periodo perinatal (Enătescu et al., 2020). A la luz de este drama existencial, se puede pensar el suicidio de las madres como una huida del sufrimiento o como una autoinculpación por no lograr el ideal materno socialmente prescrito.

Siendo así, se reclama la necesidad de una intervención preventiva profesional en el periodo perinatal. Pero no bastaría con atender sin entender, sabido que sin comprender lo que se activa es la explicación del modelo biomédico (Pérez-Álvarez, 2019). Se precisa ayudar a las madres (y a sus parejas) a elaborar el significado de su experiencia desde una perspectiva más amplia, a manejarse más eficazmente con el malestar, a integrar las diferentes identidades emergentes y a recuperar la autoría y continuidad de su vida. La ayuda psicológica debería llevarse a cabo sin caer en el error de pensar que ahí existe una "enfermedad" mental. Los profesionales de la psicología debemos permanecer atentos a las consecuencias iatrogénicas en la identidad y la autoestima que tienen las atribuciones internas, estables y globales que con frecuencia se practican para dar cuenta de las experiencias problemáticas.

\section{CONTRA EL TABÚ: SENSIBILIZACIÓN, FORMACIÓN Y CIENCIA}

Pongamos los datos encima de la mesa: parece que hay consenso entre los profesionales e investigadores en que durante el período perinatal puede haber amplias fluctuaciones en el estado de ánimo que pueden requerir atención inmediata y urgente para prevenir el riesgo de suicidio o filicidio (Kimmel, 2020; Rodríguez-Muñoz, 2019). Además, contrariamente a la idea errónea de que el embarazo podría tener un efecto protector contra la conducta de suicidio, un creciente cuerpo de conocimiento señala, en la actualidad, que la prevalencia de ideación suicida puede ser incluso mayor entre las mujeres embarazadas que en la población general de mujeres (Enătescu et al., 2020; Khalifeh et al., 2016). Es más, la investigación reciente sugiere que la ideación suicida es un fenómeno relativamente común del embarazo en todo el mundo, con una prevalencia estimada del 5\%-20\%, aunque algunos estudios en Estados Unidos han informado de cifras hasta del 33\% (Gelaye et al., 2016). Por lo tanto, hay apoyo empírico suficiente para afirmar que las mujeres embarazadas tienen mayor ideación suicida que su correspondiente grupo de comparación en la población general, aunque los datos varían considerablemente en función de la edad y el grupo étnico (Enătescu et al., 2020; Gelaye et al., 2016). A pesar de esta elevada prevalencia, seguimos sin disponer de un modelo integral que vertebre todo el conocimiento disponible sobre la ideación suicida antes del parto. Muchos estudios se han centrado en el periodo del posparto y generalmente han limitado sus objetivos de estudio a los intentos de suicidio, y no al espectro completo de conductas suicidas. Sin embargo, la variedad de conductas suicidas en el posparto (ideación, tentativa o suicidio consumado) suelen estar precedidas por alguna expresión de la conducta suicida antes del parto. Parece que este "patrón preparto", que predice los riesgos posparto y persiste más allá del período prenatal, se observaría en el estado de ánimo materno y en la presencia de ansiedad y otros trastornos derivados del estrés (Gavin et al., 2011 ; Nock et al., 2009). Por tanto, algunos autores sostienen que el período preparto representa un período sensible y una oportunidad importante para la prevención (Onah et al., 2017). Este patrón preparto, como cualquier fenómeno psicológico, no ocurre en un vacío contextual, sino inserto en una vida donde el engranaje de los discursos dominantes, los conflictos identitarios y la falta de apoyo social juega un papel fundamental.

Dicho lo anterior y dado que -no sin cierto debate (Berman y Silverman, 2017; Chiles et al., 2019)-, la ideación suicida se considera uno de los principales predictores de los posteriores intentos de suicidio o del suicidio consumado (WHO, 2014), evaluar la ideación suicida debe constituir un asunto prioritario en la exploración clínica durante todo el periodo perinatal, así como una oportunidad de intervención para prevenir otras conductas suicidas más graves, como el paso a la acción o los actos suicidas de carácter más letal (Orsolini et al., 2016). Autores relevantes han señalado que durante la primera cita clínica debería realizarse una historia de los aspectos esenciales relacionados con la salud mental (ACOG Committe Opinion, 2018; Esscher et al., 2016; Knight et al., 2019). Si la mujer embarazada ha sido diagnosticada previamente de un trastorno afectivo grave o un trastorno psicótico, debe ser derivada a los servicios de salud mental para su seguimiento, incluso aunque en ese momento esté estable y sin tratamiento. Aquí se abre un abanico de preguntas: ¿̇quién realiza ese cribado en los servicios de ginecología y obstetricia? ¿qué profesional evalúa la presencia de problemas de salud mental en tales contextos? y, ¿qué profesionales se encargan de realizar el seguimiento de estas personas (ya sea en los servicios de salud mental o en otros contextos sanitarios)?

\section{LOS NECESARIOS FACTORES DE RIESGO Y LOS DESEABLES FACTORES DE PROTECCIÓN}

En relación con los factores de riesgo de la conducta suicida, la revisión de Gelaye et al. (2016) enumera la presencia de una historia previa de abuso infantil, violencia doméstica, embarazo no deseado, intención de aborto, falta de apoyo social, nivel académico y socioeconómico bajo, soltería, ausencia de creencias religiosas y presencia de trastorno mental. Si nos centramos específicamente en los intentos de suicidio por su relevancia como predictor para el suicido consumado, los resultados publicados sugieren que durante el embarazo el riesgo sería mayor para las mujeres jóvenes, especialmente adolescentes, solteras, con bajo nivel educativo y consumo de alcohol. No está claro si una historia previa de aborto espontáneo puede ser un factor de riesgo, ni tampoco el papel que desempeña el consumo de drogas durante el embarazo, ya que hay estudios con resultados opuestos (Gressier et al., 2017). Durante el posparto el principal factor de riesgo para un intento de suicidio sería un nivel educativo bajo, mientras que el estado civil, la edad o las complica- 
ciones obstétricas parece que no serían tan relevante. Aunque existe poca investigación al respecto, a los factores de riesgo anteriores habría que añadir, siquiera sea para su consideración, los referidos a los dilemas de identidad y las disonancias entre el ideal materno y la maternidad real (Paricio del Castillo y Polo Usaola, 2020). No obstante, aún debe mejorarse el perfil de las mujeres en riesgo (Gressier et al., 2017). En la tabla 1 se pueden consultar los factores de riesgo específicos para el suicidio durante el periodo perinatal recogidos en la revisión realizada por Orsolini et al. (2016).

Al contrario que la ideación suicida, el suicidio consumado entre las mujeres en el periodo perinatal es un evento menos frecuente que en la población general (Esscher et al., 2016; Fisher, 2016; Lega et al., 2020; Lysell et al., 2018). En general, el dato relevante es que dar a luz estaría asociado a una menor tasa de suicidio. No obstante, y aquí empieza la complejidad, la maternidad, para muchas mujeres, está asociada a un incremento de dificultades, transiciones de rol y dilemas de identidad. Y serían éstos (y no la mera presencia de diagnósticos previos de trastorno mental) los que pueden incrementar la aparición de problemas de salud mental y del riesgo de suicidio (García-Haro et al., 2020; Lysell et al., 2018). Es decir, interesaría no tanto considerar el parto (como acontecimiento) como un periodo de alto riesgo para las mujeres con problemas de salud mental, sino analizar cómo es que la maternidad y sus conflictos acaba por hacer que algunas mujeres acaben desarrollando pro-

\begin{tabular}{|l|l|}
\hline \multicolumn{1}{|c|}{ TABLA 1 } \\
FACTORES DE RIESGO PARA LA CONDUCTA SUICIDA EN EL \\
PERIODO PERINATAL
\end{tabular}

blemas psicológicos. En todo caso, sí parece existir consenso en la comunidad científica acerca de que, a diferencia de la población general, estas mujeres tienden a elegir métodos de suicidio con una alta probabilidad letal, principalmente mediante ahorcamientos, defenestración o precipitación desde lugares altos (Khalifeh et al., 2016; Oates, 2003). ¿ Quizás esta caracterísitica diferencial debería darnos una pista acerca del grado de desesperanza presente en estas mujeres?

En relación con los factores de protección, no se ha descrito ninguno que sea específico para el periodo perinatal, más allá de los conocidos para la población general: relaciones personales sólidas con apoyo social, emocional y financiero, creencias religiosas o espirituales, estabilidad emocional, bienestar, autoestima y optimismo, habilidades personales (resolución de problemas y solución de conflictos), hijos pequeños a los que cuidar, estilo de vida saludable, acceso a intervenciones clínicas y apoyo en la búsqueda de ayuda profesional (Turecki y Brend, 2016; Turecki et al., 2019; WHO, 2014).

Estos factores, solos o en combinación, pueden influir en el aumento o la disminución del riesgo de suicido y deben manejarse durante la intervención clínica. Tradicionalmente se ha dado más importancia al estudio de los factores de riesgo que a los de protección, desaprovechando, quizás, la posibilidad de anclarse a aspectos importantes como el sentimiento de pertenencia o el sentido de la vida. Es importante considerar y potenciar estos factores que han demostrado aumentar la capacidad de recuperación de las dificultades y de "conexión con la vida" (Al-Halabí y García-Haro, 2021). La investigación futura tendrá que discernir cuáles de estos factores son clave en el proceso suicida de las mujeres durante el periodo perinatal y cuál es el sentido de su funcionamiento. Solo así podrán elaborarse intervenciones preventivas eficaces, efectivas y eficientes (Fonseca-Pedrero et al., 2021).

\section{¿QUIÉN, CÓMO, CUÁNDO Y DÓNDE SE EVALÚA? NO HAY RESPUESTAS SATISFACTORIAS}

A pesar de contar con instrumentos específicos de evaluación de la conducta suicida es infrecuente realizar un cribado específico en las mujeres en el periodo perinatal debido, principalmente, a limitaciones de tiempo en los servicios de salud, la falta de formación en suicidio de los profesionales implicados en el cuidado perinatal y la falta de colaboración entre ginecólogos, pediatras y psicólogos (Rodríguez-Muñoz, 2019). De hecho, la ideación suicida se suele evaluar junto con el cribado de la depresión a través del ítem 10 de la Escala de Depresión Posparto de Edimburgo o el Cuestionario sobre la Salud del Paciente, en lugar de utilizar herramientas específicamente diseñadas para tal fin (Muñiz et al., 2020). Además, muchas mujeres con ideación suicida durante el embarazo y el posparto no cumplen criterios para un diagnóstico de depresión y pueden no ser detectadas adecuadamente. Más aún, varios autores han señalado que no está clara la relevancia clínica del ítem 10 previamente mencionado para la detección de estos pensamientos y que en muchas ocasiones genera más preocupación que utilidad, ya que muchos profesionales no saben cómo abordar la pregunta ni la posible respuesta, o cómo interpretar la "normalidad" de tener pensamientos 
suicidas (Berman y Silverman, 2017; Howard et al., 2011 1). Así, muchos de ellos temen hacer estas preguntas por miedo a inducir este tipo de pensamientos, lo que evidencia una preocupante existencia de mitos sobre el suicidio entre los profesionales del Sistema Nacional de Salud (Stanley et al., 2020). Por lo tanto, reivindicamos aquí la necesaria presencia de la entrevista clínica y del entrenamiento y la formación de todos los profesionales implicados en la asistencia sanitaria de las futuras o recientes madres (Al-Halabí y García-Haro, 2021; Rodríguez-Muñoz, 2019). Orsolini et al. (2016) plantean incluir un cribado y una evaluación cuidadosa (y preventiva) de la ideación suicida durante todo el periodo perinatal (véase tabla 2). Para ello, a pesar de las dificultades señaladas previamente, proponen instrumentos específicos como la Escala de Ideación Suicida de Beck, la Escala Columbia para Evaluar el Riesgo de Suicidio o la Escala de Probabilidad de Suicidio (Al-Halabí et al., 2016; Orsolini et al., 2016).

No obstante, la evaluación a través de cuestionarios autoinformados no está exenta de limitaciones (Berman y Silverman, 2017; Vourilehto et al., 2014). Además, no podemos olvidar el carácter fluctuante de la conducta suicida, que puede no estar presente durante el cribado, desterrando su necesario seguimiento durante todo el periodo perinatal. Para advertir tal extremo, es necesario saber cómo "funcionan" las crisis suicidas, así como contar con modelos dinámicos, sistemas de evaluación ambulatoria y propuestas generales de intervención (Fonseca-Pedrero et al., 2020; Labouliere et al., 2018). De nuevo, la presencia de mitos entre los profesionales de la salud supone una enorme barrera para el adecuado apoyo y seguimiento de estas mujeres. Los cuestionarios autoadministrados son un complemento ideal de la entrevista, pero nunca han de sustituirla. Por ese motivo, volvemos a poner de manifiesto la importancia de la psicología perinatal. La idea implícita, vivida por muchas mujeres como mandato social, de que deberían encontrarse en una de las etapas más felices de su vida puede forjar una enorme culpa y vergüenza que impida expresar sus dudas, sus miedos y sus ideas de muerte. Por lo tanto, es vital que haya un profesional de la psicología delante de esa mujer con ideación suicida, manejando las emociones. Nunca se insistirá lo suficiente en señalar la importancia de las habilidades de la entrevista terapéutica en la evaluación y ayuda a las personas en riesgo suicida (Al-Halabí y García-Haro, 2021). Esto se debe a la propia naturaleza dinámica, interactiva, contextual y existencial del fenómeno suicida. En la crisis suicida hay una gran ambivalencia o conflicto dilemático entre permanecer en la vida y decidir la muerte. Por un lado, las mujeres que luchan contra el acoso de las ideas suicidas pueden sentir que han perdido las riendas de su vida, que se han equivocado en su decisión de ser madres y que ya nada volverá a ser igual. La idea es tan devastadora, que un periodo de llanto continuo del bebé o la obligación de tener que bañarlo puede fulminar un precario equilibrio de la madre, que ya no puede ver más allá de su malestar, de los efectos de su prolongada privación de sueño (Gelaye et al., 2017), y de los cambios en su cotidianidad, en su cuerpo, en su trabajo, en su pareja, en su sexualidad, en definitiva, en su vida y en su identidad. En palabras de Chiles et al. (2019), estas mujeres estarían atrapadas en lo que denominan las "tres ĺes", un dolor que ellas consideran Inescapable,
Intolerable e Interminable. Pero, como se dijo anteriormente, más allá de la presencia de un problema psicológico o de la presencia de un trastorno mental, la maternidad entraña dificultades, renuncias y nuevas identidades que pueden resultar conflictivas para algunas mujeres. Es necesario que el psicólogo profesional valide su sufrimiento, cuestionando la consideración social de la maternidad como una etapa "necesariamente" feliz. Ello no significa que se renuncie a la posibilidad de una maternidad gozosa, sobre todo en un tiempo en el que es ya una opción y no un destino.

En todo caso, la estimación del riesgo del suicidio debe hacerse en colaboración con la mujer concreta a la que se está evaluando, considerando las vivencias particulares de su maternidad y escuchando atentamente las razones que le lleva a contemplar el suicidio en vez de la vida (NICE, 2020). Además, siempre se debe evaluar la capacidad para cuidar de su hijo, así como cualquier pensamiento de daño al bebé. Las directrices establecidas por The Centre of Perinatal Excellence (COPE) (Austin et al., 2017) pueden consultarse en la tabla 3.

Una vez que el riesgo de suicidio ha sido identificado, Austin et al. (2017) proponen las siguientes consideraciones adicionales:

$\checkmark$ Riesgo bajo: analizar qué eventos precipitan los pensamientos fugaces de suicidio. Si los factores desencadenantes son inherentes a la experiencia actual con la maternidad (por ejemplo, vergüenza por los pensamientos negativos hacia el bebé), es necesario elaborar un plan de seguridad (Stanley y Brown, 2012).

\section{TABLA 2 \\ EVALUACIÓN DEL RIESGO DE CONDUCTA SUICIDA EN EL PERIODO PERINATAL}

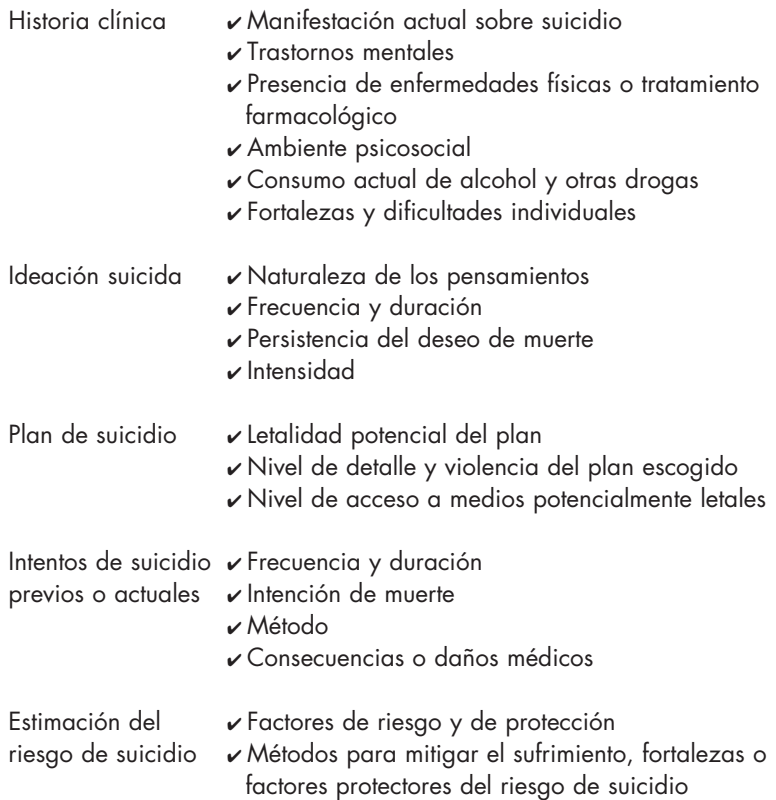

Historia clínica

$\checkmark$ Manifestación actual sobre suicidio

$\checkmark$ Trastornos mentales

$\checkmark$ Presencia de enfermedades físicas o tratamiento farmacológico

$\checkmark$ Ambiente psicosocial

$\checkmark$ Consumo actual de alcohol y otras drogas

$\checkmark$ Fortalezas y dificultades individuales

Ideación suicida $\quad \checkmark$ Naturaleza de los pensamientos

$\checkmark$ Frecuencia y duración

$\checkmark$ Persistencia del deseo de muerte

$\checkmark$ Intensidad

Plan de suicidio

$\checkmark$ Letalidad potencial del plan

$\checkmark$ Nivel de detalle y violencia del plan escogido

$\checkmark$ Nivel de acceso a medios potencialmente letales

Intentos de suicidio $\checkmark$ Frecuencia y duración

previos o actuales $\boldsymbol{v}$ Intención de muerte

$\checkmark$ Método

$\checkmark$ Consecuencias o daños médicos

Estimación del $\quad \checkmark$ Factores de riesgo y de protección

riesgo de suicidio $\checkmark$ Métodos para mitigar el sufrimiento, fortalezas o factores protectores del riesgo de suicidio

Nota. Adaptado de Orsolini et al. (2016) 
$\checkmark$ Riesgo medio: evaluar el contexto general en el que tienen lugar los pensamientos de suicidio (por ejemplo, tentativas previas de suicidio) y establecer los factores que pueden contribuir a la escalada del riesgo (por ejemplo, el llanto del bebé o una discusión). Es necesario contar con personas que puedan cuidar del bebé y la madre.

$\checkmark$ Riesgo alto: buscar una persona de apoyo que cuide del bebé. La madre puede negar la intención de suicidarse, pero correr un alto riesgo. Una mujer con un deterioro significativo de su salud mental, incapacidad para dormir, pensamientos distorsionados, incapacidad para cuidarse a sí misma o al bebé, etc., puede tener el mismo riesgo que una mujer con intención declarada de suicidio.

\section{TIEMPOS DE CAMBIO}

Abordar y prevenir de forma explícita el riesgo de suicido de estas mujeres es fundamental para reducir las tasas de suicidio en esta población que, como hemos visto, representan la principal causa de muerte durante el periodo perinatal en los países desarrollados (Enătescu et al., 2020). Algunas intervenciones preventivas con apoyo empírico se están realizando en otros países con resultados muy prometedores (Kimmel, 2020). En España se cuenta con algunas iniciativas centradas en la depresión perinatal que podrían constituir un modelo para el abordaje de factores de riesgo del suicidio (MarcosNajera et al., 2017). Rodríguez-Muñoz et al. (2017) han puesto de manifiesto que, siguiendo las recomendaciones de The American College of Obstetricians and Gynecologists (ACOG Committe Opinion, 2018 ), es posible llevar a cabo rutinas de salud y cribados para problemas de salud mental en un entorno hospitalario público y en colaboración con los servicios de obstetricia. Por supuesto, cualquier iniciativa de prevención pasaría por comenzar con una evaluación y un análisis detallados del fenómeno, algo de lo que, por el momento, aún no disponemos. Así mismo, habría que enfrentar las dificultades de la falta de tiempo en las consultas y la ausencia de unidades especializadas, gestionar los recursos disponibles y construir programas o proponer intervenciones apoyadas empíricamente. Es necesario resaltar la necesidad de utilizar tratamientos psicológicos apoyados empíricamente (Fonseca-Pedrero et al., 2021; Rodríguez-Muñoz y Al-Halabí, 2020). No es objeto de este artículo desarrollar este aspecto (para información detallada puede consultarse Al-Halabí y García Haro, 2021 y Al-Halabí et al., 2021), no obstante, a modo de resumen, los estudios revisados apoyan la eficacia de la Terapia Cognitivo Conductual y de la Terapia Dialéctico Conductual. Este tipo de terapias ofrecen la oportunidad de debatir acerca de problemas existenciales en un ambiente seguro, donde los psicólogos pueden validar el sufrimiento de las mujeres con deseos de morir o que luchan por permanecer vivas a la vez que se reorientan hacia la vida con nuevas estrategias de afrontamiento. Además, se ha desarrollado un cuerpo de intervenciones breves para dar respuesta a las emergencias clínicas de las personas en situación de crisis suicidas. Los contactos de apoyo y seguimiento o el Plan de Seguridad de Stanley y Brown son algunas de las intervenciones más efectivas y pueden combinarse con otro tipo de terapias más comprehensivas (Al-Halabí y García Haro, 202 1; AlHalabí et al., 2021).

\section{RECAPITULACIÓN}

Los problemas de salud mental durante el periodo perinatal suelen requerir de atención urgente debido a las potenciales consecuencias sobre el bienestar del bebé y la propia vida de la mujer. No obstan-

\begin{tabular}{|c|c|c|}
\hline \multicolumn{3}{|c|}{$\begin{array}{c}\text { TABLA } 3 \\
\text { PRINCIPIOS GENERALES DE ACTUACIÓN }\end{array}$} \\
\hline \multicolumn{3}{|c|}{$\begin{array}{c}\text { PREGUNTAR } \\
\text { Ideas de suicidio - Plan - Letalidad - Medios - Historia previa }\end{array}$} \\
\hline $\begin{array}{l}\text { Pensamientos fluctuantes de suicidio o autolesión } \\
\text { sin planes ni medios letales. }\end{array}$ & $\begin{array}{c}\text { Pensamientos e intenciones de suicidio sin } \\
\text { planes. }\end{array}$ & $\begin{array}{l}\text { Pensamientos continuos de suicidio, intención, } \\
\text { plan y medios. }\end{array}$ \\
\hline Riesgo bajo & Riesgo medio & Riesgo alto \\
\hline $\begin{array}{l}\text { Analizar la disponibilidad de apoyo y las } \\
\text { opciones de tratamiento. }\end{array}$ & $\begin{array}{l}\text { Analizar la disponibilidad de apoyo y las } \\
\text { opciones de tratamiento. Establecer un } \\
\text { seguimiento semanal. }\end{array}$ & $\begin{array}{c}\text { Cerciorarse de la seguridad de la mujer (ambiente } \\
\text { controlado y seguro). }\end{array}$ \\
\hline $\begin{array}{l}\text { Organizar un seguimiento y supervisión } \\
\text { adecuadas de acuerdo al juicio clínico. }\end{array}$ & $\begin{array}{l}\text { Realizar un plan de contingencia por si la } \\
\text { situación empeora y hay una escalada "suicida". }\end{array}$ & $\begin{array}{l}\text { Establecer un seguimiento en las próximas } 24 \\
\text { horas con supervisión y evaluación continua. }\end{array}$ \\
\hline $\begin{array}{l}\text { Proporcionar el contacto de recursos } \\
\text { comunitarios. }\end{array}$ & Realizar un plan de seguridad. & Controlar el riesgo para el bebé. \\
\hline $\begin{array}{l}\text { Nota. }{ }^{*} \text { Considerar siempre la salud mental de la madre y e } \\
\text { Adaptado de Austin et al. (2017) }\end{array}$ & o para el bebé. & \\
\hline
\end{tabular}


te, este tipo de problemas no suelen reconocerse adecuadamente. Muchas mujeres no buscan ayuda por miedo al estigma o a la intervención de los servicios sociales. Además, la propia maternidad puede suponer una barrera, ya que las demandas del bebé pueden interferir con la posibilidad de asistir regularmente a las sesiones de tratamiento. También pueden estar presentes otras murallas, como los problemas financieros, el miedo a tener que interrumpir la lactancia, las prioridades mal administradas o la falta de apoyo de la pareja y de la familia. Sin embargo, si las disonancias y dilemas asociados a la maternidad, así como los problemas de salud mental, no se tratan, éstos pueden afectar al bienestar de las mujeres, al de sus hijos y al de otros miembros de la familia (Al-Halabí et al., 2019; Rodríguez-Muñoz, 2019). Hacemos nuestra la máxima de "más vale un gramo de prevención que un kilo de curación", especialmente ante desenlaces que suponen la pérdida de la vida de personas debido a un dolor psicológico que no pueden tolerar.

Para finalizar: es necesario contar con modelos comprehensivos de carácter psicológico, centrados en estas mujeres y en sus experiencias con la maternidad. De nuevo, reclamamos la presencia estructural del profesional de la psicología en las plantillas y unidades de ginecología y obstetricia para una buena salud mental en la etapa perinatal, tanto de las madres, como de sus bebés en el futuro. Entendemos que prevenir los problemas en las madres es dar una buena salud psicológica a sus hijos. De otra manera, ¿̇qué relación afectiva establece una madre con ideación suicida con su bebé? No pretendemos dar respuesta a esta pregunta, pero sí queremos invitar a la reflexión y subrayar de nuevo que las ventajas de la prevención van más allá de la mera salud de las madres. Los profesionales de la psicología deben estar formados y preparados para liderar iniciativas destinadas a prevenir y abordar un fenómeno que, entendemos, es genuinamente psicológico. En palabras de la directora de The Maternal Mental Health Alliance de Reino Unido, "debemos recordar a cada una de las mujeres y de las familias que hay detrás de las cifras devastadoras de muertes por suicidio y honrar su memoria abordando urgentemente las brechas en la atención de la salud mental perinatal. Para salvar vidas, debemos asegurarnos de que todas las mujeres puedan tener acceso a la atención profesional y al apoyo necesario, cuando y donde lo necesiten".

\section{AGRADECIMIENTOS}

Los autores quieren expresar su sincero agradecimiento al profesor José M. Errasti Pérez por la minuciosa revisión de este trabajo.

\section{CONFLICTO DE INTERESES}

No existe conflicto de intereses.

\section{REFERENCIAS}

ACOG Committe Opinion Number 757(2018). Screening for perinatal depression. Obstetrics \& Gynecology, 132(5), e208-e2 12. https://www.acog.org/clinical/clinical-guidance/committeeopinion/articles/2018/11/screening-for-perinatal-depression

Al-Halabí, S. (2019). Infanticidio. En M.F. Rodríguez Muñoz (Coord.). Psicología Perinatal: Teoría y Práctica (pp. 499-500). Pirámide.
Al-Halabí, S., \& García Haro, J.M. (2021). Tratamientos psicológicos para la conducta suicida. En E. Fonseca Pedrero (Coord.), Manual de tratamientos psicológicos. Adultos (pp. 639-675). Pirámide.

Al-Halabí, S., García Haro, J.M., \& Gutiérrez López, B. (2021). Tratamientos psicológicos para la conducta suicida en adolescentes. En E. Fonseca Pedrero (Coord.), Manual de tratamientos psicológicos. Infancia y adolescencia (pp. 577-615). Pirámide.

Al-Halabí, S., Mayoral, M., \& Corcoran, P. (2019). Suicidio durante el embarazo y el posparto. En M. F. Rodríguez Muñoz (Ed.), Psicología Perinatal: Teoría y Práctica (pp. 259-272). Pirámide.

Al-Halabí, S., Sáiz, P.A., Burón, P., Garrido, M., Benabarre, A., Jiménez, E., Cervilla, J., Navarrete, M.I., Díaz-Mesa, E.M., García-Álvarez, L., Muñiz, J., Posner, K., Oquendo, M.A., García-Portilla, M.P., \& Bobes, J. (2016). Validation of a Spanish version of the Columbia-Suicide Severity Rating Scale (C-SSRS). Revista de Psiquiatría y Salud Mental [Journal of Psychiatry and Mental Health, 9, 134-142. doi.org/10.1016/i.rpsm.2016.02.002

Austin, M.P, Highet, N., \& the Expert Working Group (2017). Mental health care in the perinatal period: Australian clinical practice guideline. Centre of Perinatal Excellence.

Berman, A. L., \& Silverman, M. M. (2017). How to ask about suicide? A question in need of an empirical answer. Crisis, 38(4), 213-216. doi.org/10.1027/0227-5910/a000501

Chiles, J. A., Strosahl, K. D., \& Roberts, L. W. (2019). Clinical manual for assessment and treatment of suicidal patients ( $2^{\underline{a}}$ ed.). American Psychiatric Association.

De Beurs, D., Bockting, C., Kerkhof, A., Scheepers, F., O'Connor, R. C., Penninx, B., \& van de Leemput, I. (2020) A network perspective on suicidal behavior: understanding suicidality as a complex system. Suicide and Life-Threatening Behavior, 00, 1-12. doi.org/10.1111/sltb.12676

Enătescu, I., Craina, M., Gluhovschi, A., Giurgi-Oncu, C., Hogea, L., Nussbaum, L. A., \& Enătescu, V. R. (2020). The role of personality dimensions and trait anxiety in increasing the likelihood of suicide ideation in women during the perinatal period. Journal of Psychosomatic Obstetrics and Gynecology, 1-11. doi.org/10.1080/0167482X.2020.1734790

Esscher, A., Essén, B., Innala, E., Papadopoulos, F.C., Skalkidou, A., Sundström-Poromaa, I., \& Högberg U. (2016). Suicides during pregnancy and 1 year postpartum in Sweden, 1980-2007. British Journal of Psychiatry, 208(5), 462-469. doi.org/10.1192/bjp.bp.114.161711

Fisher, J. (2016). Perinatal psychiatric care needs of women who die by suicide. The Lancet. Psychiatry, 3(3), 191-192. doi.org/10.1016/S2215-0366(16)00025-0

Fonseca Pedrero, E., Pérez-Álvarez, M., Al-Halabí, S., Inchausti, F., Muñiz, J., López-Navarro, E., Pérez de Albéniz, A., Lucas Molina, B., Debbané, M., Bobes-Bascarán, M. T., Gimeno-Peón, A., Prado-Abril, J., Fernández-Álvarez, J., Rodríguez-Testal, J. F., González Pando, D., Díez-Gómez, A., García Montes, J. M., García-Cerdán, L., Osma, J., Peris Baquero, Ó., ... Marrero, R. J. (2021). Tratamientos psicológicos empíricamente apoyados para adultos: Una revisión selective [Evidence-Based Psychological 
Treatments for Adults: A Selective Review]. Psicothema, 33(2), 188-197. doi.org/10.7334/psicothema2020.426

Fonseca-Pedrero, E., Díez-Gómez, A., de la Barrera, U., SebastianEnesco, C., Ortuño-Sierra, J., Montoya-Castilla, I., Lucas-Molina, B., Inchausti, F., \& Pérez-Albéniz, A. (2020). Suicidal behaviour in adolescents: A network analysis. Revista de Psiquiatria y Salud Mental [Journal of Psychiatry and Mental Health]. /doi.org/10.1016/i.rpsm.2020.04.007

García-Haro, J., García-Pascual, H., \& González González, M. (2018). Un enfoque contextual-fenomenológico sobre el suicidio. Revista de la Asociación Española de Neuropsiquiatría, 38, 381 400.

García-Haro, J. M., García-Pascual, H., González González, M., Barrio-Martínez, S., \& García-Pascual, R. (2020). Suicidio y trastorno mental: Una crítica necesaria. Papeles del Psicólogo, 41 , 35-42. doi.org/10.23923/pap.psicol2020.2919

Gavin, A. R., Tabb, K. M., Melville, J. L., Guo, Y., \& Katon, W. (2011). Prevalence and correlates of suicidal ideation during pregnancy. Archives of Women's Mental Health, 14(3), 239 246. doi.org/10.1007/s00737-01 1-0207-5

Gelaye, B., Addae, G., Neway, B., Larrabure-Torrealva, G.T., Qiu, C., Stoner, L., Luque Fernandez, M.A., Sanchez, S.E., \& Williams, M.A. (2017). Poor sleep quality, antepartum depression and suicidal ideation among pregnant women. Journal of Affective Disorders, 209, 195-200. doi.org/10.1016/i.jad.2016.11.020

Gelaye, B., Kajeepeta, S., \& Williams, M.A. (2016). Suicidal ideation in pregnancy: An epidemiologic review. Archives of Women's Mental Health, 19,741-751.

Gressier, F., Guillard, V., Cazas, O., Falissard, B., Glangeaud-Freudenthal, N. M., \& Sutter-Dallay, A. L. (2017). Risk factors for suicide attempt in pregnancy and the post-partum period in women with serious mental illnesses. Journal of Psychiatry Research, 84, 284-291.

Howard, L.M., Flach, C., Mehay, A., Sharp. D., \& Tylee, A. (2011). The prevalence of suicidal ideation identified by the Edinburgh Postnatal Depression Scale in postpartum women in primary care: Findings from the RESPOND trial. BMC Pregnancy Childbirth, 11, 57. doi.org/10.1186/1471-2393-11-57

Khalifeh, H., Hunt, I.M., Appleby, L., \& Howard, L.M. (2016). Suicide in perinatal and non-perinatal women in contact with psychiatric services: 15 year findings from a UK national inquiry. The Lancet. Psychiatry, 3, 233-242. 10.1016/S2215-0366(16)00003-1

Knight, M., Bunch, K., Tuffnell, D., Shakespeare, J., Kotnis, R., Kenyon, S., \& Kurinczuk, J.J. (Eds.). (2019). Saving lives, improving mothers' care - Lessons learned to inform maternity care from the UK and Ireland confidential enquiries into maternal deaths and morbidity 2015-17. National Perinatal Epidemiology Unit, University of Oxford.

Hill, N., Robinson, J., Pirkis, J., Andriessen, K., Krysinska, K., Payne, A., Boland, A., Clarke, A., Milner, A., Witt, K., Krohn, S., \& Lampit, A. (2020). Association of suicidal behavior with exposure to suicide and suicide attempt: A systematic review and multilevel meta-analysis. PLoS Medicine, 17(3), e 1003074. doi.org/10.1371/journal.pmed.1003074
Kimmel, M. (2020). Maternal mental health matters. North Carolina Medical Journal, 81, 45-50.

Labouliere, C. D., Vasan, P., Kramer, A., Brown, G., Green, K., Rahman, M., Kammer, J., Finnerty, M., \& Stanley, B. (2018). "Zero Suicide" - A model for reducing suicide in United States behavioral healthcare. Suicidology, 23(1), 22-30.

Leather, J. Z., O'Connor, R. C., Quinlivan, L., Kapur, N., Campbell, S., \& Armitage, C. J. (2020). Healthcare professionals' implementation of national guidelines with patients who self-harm. Journal of Psychiatric Research, 130, 405-411. doi.org/10.1016/i.jpsychires.2020.08.031

Lega, I., Maraschini, A., D’Aloja, P., Andreozzi, S., Spettoli, D., Giangreco, M., Vichi, M., Loghi, M., Donati, S., \& Regional maternal mortality working group (2020). Maternal suicide in Italy. Archives of Women's Mental Health, 23(2), 199-206. 10.1007/s00737-019-00977-1

Lindahl, V., Pearson, J. L., \& Colpe, L. (2005). Prevalence of suicidality during pregnancy and the postpartum. Archives of Women's Mental Health, 8(2), 77-87. doi.org/10.1007/s00737-0050080- 1

Lysell, H., Dahlin, M., Viktorin, A., Ljungberg, E., D'Onofrio, B. M., Dickman, P., \& Runeson, B. (2018). Maternal suicide - Register based study of all suicides occurring after delivery in Sweden 1974-2009. PLoS One, 13, e0190133.

Marcos-Nájera, R. Rodríguez Muñoz, M.F., Olivares, M.E., \& Soto Balbuena C. (2017). Depresión perinatal: Rentabilidad y expectativas de la intervención preventiva. Clínica y Salud, 28, 49-52.

Martini, J., Baver, M., Lewitzka, U., Voss, C., Pfennig, A., Ritter, D., \& Wittchen, H. U. (2019). Predictors and outcomes of suicidal ideation during peripartum period. Journal of Affective Disorders, 257, 518-526.

Muñiz, J., Hernández, A., \& Fernández-Hermida, J.R (2020). Utilización de los test en España: El punto de vista de los psicólogos. Papeles del Psicólogo, 4 1(1), 1-15. doi:10.23923/pap.psicol2020.2921

NICE, National Institute for Health and Care Excellence (2020). Antenatal and postnatal mental health: Clinical management and service guidance (CG 192). https://www.nice.org.uk/guidan$\mathrm{ce} / \mathrm{cg} 192$

Nock, M. K., Hwang, I., Sampson, N., Kessler, R. C., Angermeyer, M., Beautrais, A., Borges, G., Bromet, E., Bruffaerts, R., de Girolamo, G., de Graaf, R., Florescu, S., Gureje, O., Haro, J. M., Hu, C., Huang, Y., Karam, E. G., Kawakami, N., Kovess, V., Levinson, D., ... Williams, D. R. (2009). Cross-national analysis of the associations among mental disorders and suicidal behavior: findings from the WHO World Mental Health Surveys. PLoS Medicine, 6(8), e1000123. doi.org/10.1371/journal.pmed.1000123

Oates, M. (2003). Suicide: the leading cause of maternal death. British Journal of Psychiatry, 183(4), 279-281. doi.org/10.1192/bip.183.4.279

Onah, M. N., Field, S., Bantjes, J., \& Honikman, S. (2017). Perinatal suicidal ideation and behaviour: Psychiatry and adversity. Archives of Women's Mental Health, 20(2), 321-331. doi.org/10.1007/s00737-016-0706-5

Orsolini, L., Valchera, A., Vecchiotti, R., Tomasetti, C., lasevoli, F., 
Fornaro, M., De Berardis, D., Perna, G., Pompili, M., \& Bellantuono, C. (2016). Suicide during perinatal period: Epidemiology, risk factors, and clinical correlates. Frontiers in Psychiatry, 7, 138. doi.org/10.3389/fpsyt.2016.00138

Paricio del Castillo, R., \& Polo Usaola, P. (2020). Maternidad e identidad materna: deconstrucción terapéutica de narrativas. Revista de la Asociación Española de Neuropsiquiatría, 40(138), 33-54.

Pérez-Álvarez, M. (2019). La psicoterapia como ciencia humana, más que tecnológica. Papeles del Psicólogo, 40(1), 1-14. doi.org/10.23923/pap.psicol2019.2877

Rodríguez-Muñoz, M.F., \& Al-Halabí, S. (2020). A pathway to excellence. Clínica y Salud, 31, 125-126. doi.org/10.5093/clysa2020a3 1

Rodríguez-Muñoz, M. (2019). Psicología perinatal: Teoría y práctica. Pirámide.

Rodríguez-Muñoz, M., Le, H. N., de la Cruz, I. V., Crespo, M., \& Méndez, N. I. (2017). Feasibility of screening and prevalence of prenatal depression in an obstetric setting in Spain. European Journal of Obstetrics, Gynecology, and Reproductive Biology, 215, 101-105. doi.org/10.1016/j.ejogrb.2017.06.009

Rodríguez-Muñoz, M.F., Soto-Balbuena, C., Olivares Crespo, M. E., Marcos-Nájera, R., \& Al-Halabí, S. (2021). Tratamientos psicológicos para los trastornos mentales o del comportamiento asociados con el embarazo, el parto o el puerperio. En E. Fonseca Pedrero (Coord.), Manual de tratamientos psicológicos. Adultos [Psychological treatment Manual. Adults] (pp. 607-639). Pirámide.

Rendueles, G. (2018). Suicidio(s) [Suicide(s)]. Grupo 5 [Group 5].

Silverman, M. M. (2006). The language of suicidology. Suicide and life-threatening behavior, 36(5), 519-532.

Silverman, M. M. (2016). Challenges to defining and classifying suicide and suicidal behaviours. In R. O'Connor \& J. Pirkis (Eds.), International handbook of suicide prevention (2nd ed.) (pp. 11-35). Wiley Blackwell.

Silverman, M. M., \& Berman, A. L. (2014). Suicide risk assessment and risk formulation Part I: A focus on suicide ideation in assessing suicide risk. Suicide and Life-Threatening Behavior, 44(4), 420431.
Silverman, M. M., \& Deleo, D. D. (2016). Why there is a need for an international nomenclature and classification system for suicide. Crisis, 37(2), 83-87.

Stanley, B., \& Brown, G.K. (2012). Safety planning intervention: A brief intervention to mitigate suicide risk. Cognitive and Behavioral Practice, 19(2), 256-264. doi.org/10.1016/i.cbpra.2011.01.001

Stanley, B., Labouliere, C. D., Brown, G. K., Green, K. L., Galfalvy, H. C., Finnerty, M. T., Vasan, P., Cummings, A. K., Wainberg, M., Carruthers, J. W., \& Dixon, L. B. (2020). Zero suicide implementation-effectiveness trial study protocol in outpatient behavioral health using the A-I-M suicide prevention model. Contemporary Clinical Trials, 100, 106224. doi.org/10.1016/i.cct.2020.106224

Turecki, G., \& Brent D.A. (2016). Suicide and suicidal behaviour. Lancet, 387, 1227-12239.

Turecki, G., Brent, D.A., Gunnell, D., O'Connor, R. C., Oquendo, M. A., Pirkis, J., \& Stanley, B.H. (2019). Suicide and suicide risk. Nature Reviews Disease Primers, 5, 74.

van Mens, K., de Schepper, C., Wijnen, B., Koldijk, S. J., Schnack, H., de Looff, P., Lokkerbol, J., Wetherall, K., Cleare, S., C O'Connor, R., \& de Beurs, D. (2020). Predicting future suicidal behaviour in young adults, with different machine learning techniques: A population-based longitudinal study. Journal of Affective Disorders, 271, 169-177. doi.org/10.1016/i.jad.2020.03.081

Vourilehto, M., Valtonen, H. M., Melartin, T., Sokero, P., Souminen, K., \& Isometsä, E. T. (2014). Method of assessment determines prevalence of suicide ideation among patients with depression. European Psychiatry, 29, 338-344.

World Health Organization (2014). Preventing suicide: A global imperative. WHO Library.

Zalsman, G., Hawton, K., Wasserman, D., van Heeringen, K., Arensman, E., Sarchiapone, M., Carli, V., Höschl, C., Barzilay, R., Balazs, J., Purebl, G., Kahn, J. P., Sáiz, P. A., Lipsicas, C. B., Bobes, J., Cozman, D., Hegerl, U., \& Zohar, J. (2016). Suicide prevention strategies revisited: 10-year systematic review. The Lancet. Psychiatry, 3(7), 646-659. doi.org/10.1016/S22150366(16)30030-X 